\title{
En homenaje a las contribuciones de Paul R. Pintrich a la investigación sobre Psicología y Educación
}

Número monográfico en homenaje a Paul R. Pintrich

Editado por Margarita Limón

Departamento de Psicología Básica, Universidad Autónoma de Madrid

España 


\section{Indice}

- En homenaje a las contribuciones de Paul R. Pintrich a la investigación sobre Psicología y Educación: introducción, Margarita Limón Luque ....................... 159-162

- Paul R. Pintrich y la investigación sobre creencias epistemo-

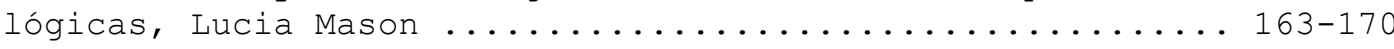

- Contribuciones de Paul R. Pintrich a la investigación sobre cambio conceptual, Gale M. Sinatra.................171-174

- Cambio conceptual y el aprendiz intencional esbozado por Paul R. Pintrich, Margarita Limón................... 175-184

- Identificando retos en la investigación sobre aprendizaje autorregulado: aportaciones de Paul R.Pintrich, Philip H.Winne. 185-188

- Sobre la obra de Paul R. Pintrich: la autorregulación de los procesos cognitivos y motivacionales en el contexto educativo, Ignacio Montero y María José de Dios............. 189-196

- El factor humano, Patricia A. Alexander................. 197-202

- La dimensión internacional de Paul R.Pintrich, Erik De Corte.. 203-206

- En homenaje a Paul R. Pintrich, Richard E. Mayer .......... 207-210 


\title{
En homenaje a las contribuciones de Paul R. Pintrich a la investigación sobre Psicología y Educación: Introducción
}

\author{
Margarita Limón
}

Departamento de Psicología Básica, Universidad Autónoma de Madrid, Madrid

\section{España}

margarita.limon@uam.es

\begin{abstract}
Resumen
Esta parte del monográfico presenta los comentarios de varios especialistas internacionales sobre las contribuciones de Paul R. Pintrich a la investigación sobre Psicología y Educación. Lucia Mason (Universidad de Padua, Italia) comenta las aportaciones del trabajo del profesor Pintrich y sus colaboradores sobre las creencias epistemológicas y su papel en el aprendizaje y la enseñanza. Gale Sinatra (Universidad de Nevada, USA) y Margarita Limón (Universidad Autónoma de Madrid, España) respectivamente, ofrecen su visión de las contribuciones de Paul a la investigación sobre cambio conceptual. Philip Winne (Simon Fraser University, Canadá) por un lado, e Ignacio Montero y $M^{a}$ José de Dios (Universidad Autónoma de Madrid, España), por otro, analizan las aportaciones tanto teóricas como empíricas de Paul Pintrich en el ámbito del aprendizaje autorregulado y la motivación en contextos académicos.

Finalmente, Richard E. Mayer (Universidad de California en Santa Bárbara, USA), Patricia A. Alexander (Universidad de Maryland, USA) y Erik De Corte (Universidad de Lovaina, Bélgica) presentan un comentario general sobre las contribuciones y repercusiones de la obra de Paul Pintrich en Psicología y Educación.
\end{abstract}

Palabras clave: Creencias epistemológicas / Cambio conceptual/ Motivación / Aprendizaje autorregulado/ Psicología Educativa / Psicología Cognitiva 


\section{Introducción}

En esta parte de este número monográfico en homenaje a nuestro querido amigo y compañero Paul R. Pintrich, ofrecemos a los lectores un breve comentario de conocidos especialistas internacionales sobre cuatro de los temas en los que se centró su investigación: las creencias epistemológicas y su papel en el aprendizaje y la enseñanza, cambio conceptual, motivación en contextos académicos y autorregulación del aprendizaje.

No obstante, como puede apreciarse en la pequeña biografía que acompaña a esta introducción, la brillante trayectoria académica de Paul demuestra que su influencia en el ámbito de la Psicología y la Educación ha sido sobresaliente, no sólo a través de su trabajo de investigación, sino también gracias a su extenso trabajo como editor, revisor y miembro del Comité Editorial de prácticamente todas las revistas mayor prestigio en el campo, como Presidente de la División 15 (Educational Psychology) de la American Psychological Association (APA), de la División 5 (Educational, Instructional and School Psychology) de la International Association of Applied Psychology (IAAP), miembro destacado de la European Association for Research on Learning and Instruction (EARLI) o, como a él más le gustaba, como formador de nuevos especialistas y apoyando activa e intensamente la colaboración e intercambio de experiencias entre colegas, tanto dentro de su país como a nivel internacional.

Por todo ello, he pedido también a tres de los especialistas más destacados en el ámbito de la Psicología y la Educación, Patricia A. Alexander, Erik de Corte y Richard E. Mayer, que escribieran para este número un comentario general sobre las contribuciones generales de Paul al ámbito de la Psicología y la Educación.

Como editora de esta parte del monográfico mi propósito es que aquellos lectores que no hayan tenido el privilegio de conocer a Paul puedan hacerse rápidamente una idea de sus líneas de investigación, de por qué han sido y son relevantes para nuestro trabajo en Psicología y Educación y de los muchos caminos que nos ha dejado abiertos para continuar lo que él empezó.

Pero además, me gustaría que el lector conozca aunque sea fugazmente a través de los comentarios de sus compañeros y amigos, no sólo sus sobresalientes capacidades profesionales, sino también sus valores y sus excepcionales cualidades como ser humano. Me parece 
imprescindible destacar su profunda honradez, sencillez y humildad. Cuidar a sus estudiantes y hacer todo lo posible para ser un buen profesor eran dos de las cosas que más le gustaban.

Creo que consiguió ser un profesor modélico a todos los niveles, y que, una vez más, logró la meta que seguro, tenía planificada desde mucho tiempo atrás. Quienes lo conocimos, sabemos que su interés en desarrollar el concepto de aprendizaje autorregulado, en el que la planificación de las metas juega un papel esencial no fue casualidad, sino que tenía mucho que ver con cómo era él.

Conociendo la modestia y sencillez que lo caracterizaban, es posible que me "regañara" por escribir todo esto sobre él, incluso por aceptar editar esta parte de este monográfíco como homenaje a su trabajo. Sin embargo, estoy convencida de que es algo absolutamente merecido y lo menos que sus compañeros podemos hacer para agradecerle su trabajo, sus contribuciones a todos los niveles y hacer notar lo mucho que lo echaremos de menos.

Quiero agradecer su participación a todos los autores que han participado en esta parte del monográfico. Sé que todos tienen la agenda completamente llena y sin embargo, han sido enormemente generosos con su tiempo y han hecho un "hueco" para poder escribir estas páginas. También sé que para algunos, muy cercanos a Paul, redactar estas páginas ha supuesto un esfuerzo adicional que les agradezco doblemente.

No quiero finalizar esta introducción sin dar también las gracias a Jesús de la Fuente, editor de la revista, y a sus colaboradores por su interés y esfuerzo en publicar este monográfico en homenaje a Paul. Algunos de ellos, como miembros del Comité organizador del Congreso Internacional sobre Psicología y Educación que ha organizado la Asociación Nacional de Psicología de la Educación y Psicopedagogía y el Departamento de Psicología Evolutiva y de la Educación de la Universidad de Almería, lo habían invitado a dictar la conferencia de apertura. Sé que Paul estaba encantado de haber recibido esta invitación y estoy segura de que le habría gustado contribuir a apoyar su iniciativa de promover y desarrollar la investigación sobre Psicología y Educación. Ojalá que este monográfico pueda también a ayudar a realizar alguna aportación en esa dirección. 


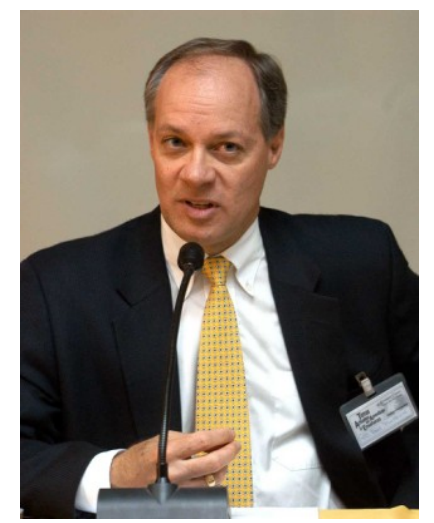

\section{Selección de algunas} de las publicaciones más recientes de Paul R. Pintrich

Pintrich, P. R. (2003). A Motivational Science Perspective on the Role of Student Motivation in Learning and Teaching Contexts. Journal of Educational Psychology, 95(4), 667686.

Sinatra, G. M., \& P. R. Pintrich (Eds.)(2003). Intentional Conceptual Change. Mahwah, NJ: Lawrence Erlbaum Associates.

Hofer, B. K., \& P. R. Pintrich (Eds.)(2002). Personal Epistemology. The Psychology of Beliefs about Knowledge and Knowing. Mahwah, NJ: Lawrence Erlbaum Associates.

Pintrich, P.R., \& Schunk, D.H. (2002). Motivation in Education (Rev. ed.). Upper Saddle River, NJ: Merrill Prentice Hall. Trad. cast. Motivación y Educación, Madrid: Pearson/ Merrill Prentice Hall, 2004.

Boekaerts, M., Pintrich, P. R., \& Zeidner, M. (Eds.)(2000). Handbook of Self-Regulation. San Diego: Academic Press.
Paul R. Pintrich era profesor de Educación y Psicología y Director del programa de Doctorado en Educación y Psicología de la Universidad de Michigan. Falleció repentinamente el 12 de Julio de 2003 cuando estaba practicando su deporte favorito, ciclismo, a causa de la rotura de la arteria aorta. Tenía 49 años.

El profesor Pintrich era un destacado especialista en Psicología de la Instrucción. Concretamente, su investigación se centró en el estudio de la motivación y su desarrollo, cambio conceptual, pensamiento epistemológico, aprendizaje autorregulado y en cómo mejorar la Educación Superior.

Fue editor de Educational Psychologist, la revista de la División 15 -Psicología Educativa- de la Asociación Americana de Psicología (APA) desde 1994 a 2000. Desde 1991, era co-editor con M. Maehr de Advances in Motivation and Achievement, JAI Press (Greenwich, Connecticut). Era miembro del Consejo Editorial del International Journal of Educational Research, Contemporary Educational Psychology, Learning and Individual Differences, American Educational Research Journal, Journal of Literacy Research, Journal of Educational Psychology (hasta 1998), Developmental Psychology (1992-1993). También era revisor y evaluador de la mayoría de las principales revistas del ámbito del Aprendizaje y la Instrucción. Hasta la fecha de su fallecimiento era el editor de las New Series on Education and Psychology publicadas por Lawrence Erlbaum Associates.

Fue presidente de la División 15-Psicología Educativa- de la Asociación Americana de Psicología en 2002. Era también presidente de la División 5 -Educational, Instructional and School Psychology de la Asociación Internacional de Psicología Aplicada (IAAP). Fue Decano (Associate Dean for Research) de la Facultad de Educación de la Universidad de Michigan desde Septiembre de 1998 a Septiembre de 2001.

Entre los muchos premios y menciones honoríficas que recibió, a él le gustaba destacar dos: el premio otorgado por la AERA al mejor artículo de revisión de investigación que ganó junto con su estudiante Bárbara Hofer en 1999 por un artículo sobre pensamiento epistemológico publicado en Review of Educational Research, y sobre todo, el premio Class of 1923 concedido en 1990 por el College of Literature, Science and Arts de la Facultad de Educación de la Universidad de Michigan como mejor profesor. Estaba muy satisfecho de este premio porque se obtenía como resultado de la votación de los estudiantes. Le encantaba tutorizar y ayudar a sus estudiantes y dirigió numerosas Tesis Doctorales.

Era miembro de la European Association for Research on Learning and Instruction (EARLI) desde 1995 y venía colaborando en múltiples actividades de esta asociación desde 1994. Sobre todo, parti-

Su familia ha creado una beca, Paul Pintrich Education and Psychology Scholarship, en homenaje a su memoria y a su dedicación hacia sus estudiantes. Este fondo permitirá que algunos estudiantes sin recursos puedan realizar estudios en Psicología y Educación. Pueden enviarse contribuciones y recibir más información en la Facultad de Educación de la Universidad de Michigan:

Laurie Stoianowski, Development Officer. University of Michigan, School of Education, 610 E. University Avenue. \#1123 B, Ann Arbor, MI 48109-1259.

E-mail: 1stoian@umich.edu Más información en: http://www.soe.umich.edu 


\title{
Paul R. Pintrich y la investigación sobre creencias epistemológicas
}

\author{
Lucia Mason \\ Dipartimento di Psicologia dello Sviluppo e della Socializzazione, \\ University of Padova
}

Italia

lucia.mason@unipd.it

\section{Resumen}

Este artículo presenta un breve comentario de la profesora Mason sobre las aportaciones de Paul R. Pintrich y sus colaboradores al campo de las creencias epistemológicas. A lo largo el mismo, la autora revisa brevemente esta área de investigación y explica qué son las creencias epistemológicas, qué papel juegan en el aprendizaje y la enseñanza y cuáles son los principales puntos de controversia en este ámbito de investigación.

Palabras claves: Creencias epistemológicas / Aprendizaje / Enseñanza 
Quiero honrar en estas páginas la memoria de Paul Pintrich destacando su excelente contribución, en colaboración con Bárbara Hofer, a la investigación psicológica sobre las creencias epistemológicas. Juntos publicaron en 1997 en el Review of Educational Research el artículo titulado "The development of epistemological theories: Beliefs about knowledge and knowing and their relation to learning" [El desarrollo de la teorías epistemológicas: creencias sobre el conocimiento y sobre cómo se adquiere y su relación con el aprendizaje], que recibió el premio al Mejor Artículo de Revisión de la AERA (American Educational Research Association) . Cuando leí este sobresaliente artículo estaba comenzando a explorar esta área de investigación y lo encontré muy ilustrativo tanto por lo completo que era, como por su riqueza, rigor y claridad.

¿Qué son las creencias epistemológicas? Son convicciones que los individuos tienen sobre el conocimiento y su adquisición, esto es, sobre la organización y las fuentes de conocimiento, su credibilidad (valor de verdad) y sobre los criterios para justificar afirmaciones, que constituyen la "epistemología personal” (Hofer y Pintrich, 1997; 2002). En la última década, la investigación sobre la epistemología personal se ha desarrollado en dos principales direcciones: el desarrollo del pensamiento epistemológico, por un lado, y los efectos de las creencias sobre el conocimiento y su adquisición en diferentes aspectos del proceso de aprendizaje, por otro (Mason, 2002). Los investigadores están de acuerdo en señalar la existencia de una transición evolutiva que conduce hacia una comprensión epistemológica madura (Moshman, 1998). En términos de Kuhn (1999, 2000; Kuhn, Cheney, \& Weinstock, 2000; Kuhn \& Weinstock, 2002), los individuos pasan de mantener una visión absolutista a una múltiple (multiplist) y de ahí a una visión evaluadora (evaluativist) del conocimiento y su adquisición.

De acuerdo con la perspectiva absolutista, el conocimiento es absoluto, cierto, no problemático, correcto o incorrecto y no necesita ser justificado, puesto que se origina a través de observaciones directas de la realidad o del jucio o afirmación emitido por una autoridad en el tema. Estas creencias caracterizan, aunque no necesariamente se dan solamente en esta etapa, - pueden aparecer también más adelante-, el pensamiento epistemológico en la infancia.

Desde la posición múltiple (multiplist) el conocimiento se concibe como ambiguo e idiosincrático, cada individuo tiene su punto de vista y su verdad. Estas creencias son típicas de la adolescencia . La perspectiva evaluadora integra y coordina la dimensión objetiva y subjetiva del conocimiento. Un individuo que sostiente un posicionamiento evaluador cree que dos personas pueden mantener puntos de vista diferentes y que ambos pueden ser "ciertos", pero uno será "más cierto" que el otro: aquel que tenga más y mejor apoyo. Esta perspectiva más sofisticada se 
desarrolla en la edad adulta y conduce a una comprensión madura de la naturaleza y justificación del conocimiento que implica procesos activos de reflexión y pensamiento crítico.

Se ha documentado empíricamente que las epistemologías personales influyen en la comprensión lectora (Schommer, 1990), en la interpretación de temas controvertidos (Kardash \& Scholes, 1996; Mason, 2000; Mason y Boscolo, 2003; Schommer (1990), en la metacomprensión (Ryan, 1984; Schommer, Crouse, \& Rhodes, 1992), en la solución de problemas mal definidos (Schraw, Dunkle, \& Bendixen,1995), en la transferencia del aprendizaje (Jacobson \& Spiro, 1995), y en el cambio conceptual (Mason, 2003; Qian \& Alvermann, 1995; Windschitl \& Andre, 2003). En todos estos estudios, las creencias epistemológicas menos avanzadas, esto es, aquellas en las que el conocimiento es considerado como absoluto, simple, estable y transmitido por una autoridad, se asocian con un rendimiento menor. Por el contrario, las creencias más sofisticadas, es decir, aquellas en las que el conocimiento es complejo, incierto, y derivado del razonamiento se asocian con un mejor rendimiento.

En su premiado artículo, Paul Pintrich y Barbara Hofer revisaban de manera global y crítica toda la investigación sobre el tema y planteaban cinco cuestiones clave pendientes de investigación. Las mencionaré brevemente.

1. Necesidad de clarificar el constructo "creencias epistemológicas", puesto que lo que se incluye o excluye bajo esa etiqueta varía, no todos los investigadores lo entienden de igual modo.

En la mayor parte de la literatura en Psicología Educativa, las creencias epistemológicas se refieren a la naturaleza del conocimiento, del aprendizaje y a menudo se incluyen las creencias sobre la inteligencia. Por otro lado, en la mayor parte de la literatura propia de la Psicología Evolutiva, el término epistemológico se refiere, en vez de al conocimiento, sólo al razonamiento y los procesos de justificación del conocimiento. Paul y Bárbara propusieron que el contenido del constructo se limitara a las creencias sobre la naturaleza del conocimiento y el proceso de adquisición del mismo y señalaron las dimensiones implicadas en él.

2. Necesidad de realizar estudios que rastreen el inicio del pensamiento epistemológico, ya que hasta entonces, eran pocos los estudios realizados en niveles anteriores a la enseñanza secundaria. En este sentido, se destaca la necesidad de conectar este campo de las creencias epistemológicas con la investigación sobre teoría de la mente emerge de este destacado y relevante artículo. Tanto los estudios sobre la teoría de la mente y la epistemología persona están interesados en el desarrollo de una teoría del conocimiento. Asimismo, investigar el desarrollo del pensamiento 
epistemológico después de la etapa universitaria, esto es, fuera de programas educativos, parece ser crucial para examinar mejor el papel del contexto sociocultural.

3. Necesidad de investigar los mecanismos motivacionales y contextuales que facilitan o limitan el cambio de epistemología personal. Por ejemplo, en contextos académicos, las prácticas de enseñanza pueden transmitir una visión del conocimiento objetivista o constructivista. Además, las relaciones entre las creencias epistemológicas de los estudiantes, su orientación motivacional, sus expectativas de autoeficacia y sus habilidades de autorregulación ponen de manifiesto la relevancia de investigar y comprender el papel de los componentes afectivos de las epistemlogías personales.

4. Necesidad de explorar las diferencias debidas al dominio en el pensamiento epistemológico, dada la naturaleza poco concluyente de los resultados obtenidos hasta entonces sobre este punto. Podría decirse, como Paul y Bárbara hipotetizan, que las creencias generales y específicas de dominio forman parte de una red de afirmaciones interconectadas sobre el conocimiento y sobre cómo se adquiere conocimiento.

5. Necesidad de utilizar y desarrollar investigación con métodos cuantitativos y cualitativos, basada tanto en el uso de instrumentos más estructurados como abiertos, puesto que la evaluación cuantitativa a gran escala debería integrarse con investigaciones de corte fenomenológico. En los primeros, los estudiantes responden a items cerrados, mientras que en los últimos generan su propio pensamiento. Sólo la combinación de estos enfoques puede conducir a una comprensión profunda de la naturaleza y el desarrollo de las creencias sobre el conocimiento y su adquisición.

Creo que Paul Pintrich, junto con Bárbara Hofer han hecho una excelente contribución a la construcción y al avance en la investigación sobre la epistemología personal. Los investigadores de esta área se han beneficiado durante un amplio período de este trabajo pionero.

Permítanme concluir estas páginas con algunos comentarios personales. Recuerdo con claridad la primera vez que me encontré a Paul. Fue en el aeropuerto de Leipzig en Alemania, en Septiembre de 1994. Los dos estábamos esperando para tomar un vuelo a Jena y participar en el primer seminario del SIG (grupo de interés especial) de "Cambio Conceptual" de EARLI (European Association for Research on Learning and Instruction). En ese momento, ya había leido 
otro trabajo sobresaliente y pionero de Paul Pintrich, junto con Marx y Boyle, "Beyond cold conceptual change: The role of motivational beliefs and classroom contextual factors in the process of conceptual change" [Más allá del cambio conceptual frio: el papel de las creencias motivacionales y de los factores contextuales de la clase en el proceso de cambio conceptual]. Me alegré de tener la oportunidad de charlar con él en un contexto informal. Tuvimos una conversación muy animada, aunque corta, sobre las diferencias culturales entre Europa y los Estados Unidos. Desde entonces, nos encontramos en otras conferencias, tanto en Europa como en Estados Unidos y nuestra colaboración fue haciéndose más cercana.

Como organizadores de la décima conferencia de EARLI en Padua, en 2003, Pietro Boscolo y yo invitamos a Paul a dar una conferencia invitada. Aceptó con entusiasmo la invitación a presentar sus resultados de investigación en una universidad antigua y famosa en el país extranjero que más le gustaba por su belleza artística, histórica y natural. Su nombre fue el primero en nuestra lista de conferenciantes invitados. Nos habriamos sentido muy honrados y orgullosos de que hubiera estado en Padua. Estuve en contacto con él hasta el dia antes de su repentina muerte.

Agradezco mucho a Paul su invitación para contribuir con un capítulo en su libro coeditado con Gale Sinatra titulado Intentional Conceptual Change, una obra muy relevante, publicada por Lawrence Erlbaum Associates al principio de 2003. Siempre me animaba a seguir adelante con mis ideas de investigación. He aprendido mucho de él y no sólo desde una perspectiva profesional. Gracias, Paul, por ser tan estimulante e inspirador y por tu apoyo cálido y cariñoso.

\section{Referencias}

Andre, T., \& Windschitl, M. (2003). Interest, Epistemological belief, and intentional conceptual change. In G.M. Sinatra \& P.R. Pintrich (Eds.), Intentional Conceptual Change (pp. 173197). Mahwah, NJ: Lawrence Erlbaum Associates.

Hofer, B.K., \& Pintrich, P.R. (1997). The development of epistemological theories: Beliefs about knowledge and knowing and their relation to learning. Review of Educational Research, 67, 88-140.

Hofer, B.K., \& Pintrich, P.R. (Eds.) (2002). Personal epistemology. The psychology of beliefs about knowledge and knowing. Mahwah, NJ: Lawrence Erlbaum Associates.

Jacobson, M.J., \& Spiro, R.J. (1995). Hypertext learning environments, cognitive flexibility, and the transfer of complex knowledge: An empirical investigation. Journal of Educational Computing Research, 12, 301-333. 
Kardash, C.M., \& Scholes, R.J. (1996). Effects of preexisting beliefs, epistemological beliefs, and need for cognition on interpretation of controversial issues. Journal of Educational Psychology, 88, 260-271.

Kuhn, D. (1999). A developmental model of critical thinking. Educational Researcher, 28, 16-26.

Kuhn, D (2000). Theory of mind, metacognition, and reasoning: A life-span perspective. In P. Mitchell \& K.J. Riggs (Eds.), Children's reasoning and the mind (pp. 301-326). Hove, UK: Psychology Press.

Kuhn, D., Cheney, R., \& Weinstock, M. (2000). The development of epistemological understanding. Cognitive Development, 15, 309-328.

Kuhn, D., \& Weinstock, M. (2002). What is epistemological thinking and why does it matter? In B.K. Hofer \& P.R. Pintrich (Eds.), Personal epistemology. The psychology of beliefs about knowledge and knowing (pp. 121-144). Mahwah, NJ: Laurence Erlbaum Associates.

Mason, L. (2000). Role of anomalous data and epistemological beliefs in middle students' theory change on two controversial topics. European Journal of Psychology of Education, 15, 329-346.

Mason, L. (2002). Developing epistemological thinking to foster conceptual changes in different domains. In M. Limón \& L. Mason (Eds.), Conceptual change reconsidered. Issues in theory and practice (pp. 301-336). Dordrecht, NL: Kluwer Academic Publishers.

Mason, L. (2003). Personal epistemologies and intentional conceptual change. In G.M. Sinatra \& P.R. Pintrich (Eds.), Intentional conceptual change (pp. 199-236). Mahwah, NJ: Lawrence Erlbaum Associates.

Mason, L. \& Boscolo, P. (2003). Role of Epistemological understanding and interest in interpreting a controversy and in topic-specific belief change. Submitted for publication.

Moshman, D. (1998). Cognitive development beyond childhood: Constraints on cognitive development and learning. In W. Damon (Series Ed.), D. Kuhn \& R. Siegler (Vol. Eds.), Handbook of child psychology: Vol. 2: Cognition, language, and perception (5th ed., pp. 947-978). New York: Wiley.

Qian, G. \& Alvermann, D. (1995). Role of epistemological beliefs and learned helplessness in secondary school students' learning of science concepts from text. Journal of Educational Psychology, 87, 282-292. 
Schommer, M. (1990). Effects of beliefs about the nature of knowledge on comprehension. Journal of Educational Psychology, 82, 498-504.

Schommer, M., Crouse, A. \& Rhodes, N. (1992). Epistemological beliefs and mathematical text comprehension: Believing it is simple does not make it so. Journal of Educational Psychology, 84, 435-443.

Schraw, G., Dunkle, M.E. \& Bendixen, L.D. (1995). Cognitive processes in well-defined and ill-defined problem solving. Applied Cognitive Psychology, 9, 523-538.

Lucia Mason es profesora de Psicología Evolutiva y de la Educación de la Facultad de Psicología de la Universidad de Padua, Italia. Actualmente, su principal línea de investigación es el estudio de la epistemología personal y sus relaciones con el proceso de aprendizaje en general, y en particular, con los procesos de cambio conceptual. Ha sido coordinadora del SIG de Cambio Conceptual de la European Association for Research on Learning and Instruction (EARLI) desde 1997 a 2001. Es co-editora de dos volúmenes publicados por Kluwer Academic Publishers Dordrecht, The Netherlands: P. Tynjala, L. Mason, \& K. Lonka (2001), Writing as a Learning Tool. Integrating Theory and Practice, y M. Limón \& L. Mason (2002), Reconsidering Conceptual Change. Issues in Theory and Practice. También es autora del libro Verità e Certezze. Natura e Sviluppo delle Epistemologie Ingenue, publicado en 2001, editorial Carocci, Roma, Italia. Es autora y coautora de numerosos artículos en revistas nacionales e internacionales y capítulos en volúmenes internacionales sobre cambio conceptual y creencias epistemológicas.

Paul y ella han participado y organizado numerosas sesiones sobre cambio conceptual y creencias epistemológicas tanto en las conferencias anuales y bianuales y reuniones de los SIGs de AERA (American Educational Research Association) y EARLI desde 1994. Ha colaborado también con Paul en dos libros recientes sobre Cambio Conceptual: Limón, M. \& Mason, L. (Eds.) (2002), Reconsidering Conceptual Change: Issues in Theory and Practice. Dordretch, The Netherlands: Kluwer Academic Press, and Sinatra, G. M. \& Pintrich, P.R. (Eds.) (2003). Intentional Conceptual Change. Mahwah, NJ: Lawrence Erlbaum Associates. 
Lucía Mason

[Página dejada en blanco intencionadamente] 


\title{
Contribuciones de Paul R. Pintrich a la investigación sobre cambio conceptual
}

\author{
Gale M. Sinatra
}

Department of Educational Psychology

University of Nevada, Las Vegas

USA

sinatra@unlv.nevada.edu

\section{Resumen}

La autora presenta su punto de vista acerca de las aportaciones de Paul R. Pintrich a la investigación sobre cambio conceptual y los cambios que él inspiró en la investigación reciente sobre este tema. Concretamente, Sinatra destaca cómo el pensamiento de Paul Pintrich y su investigación ha provocado un giro hacia modelos más "cálidos” de cambio conceptual que tienen en cuenta el papel en este proceso de las creencias motivacionales, las metas y los factores metacognitivos y sociales.

Palabras clave: Cambio Conceptual / Aprendizaje intencional 
Sin duda alguna el cambio conceptual fue el área de investigación principal de Paul Pintrich. Y lo que es más, puede decirse que su artículo de 1993, Beyond cold conceptual change: The role of motivational beliefs and classroom contextual factors in the process of conceptual change, escrito junto a Ron Marx y Robert Boyle, cambió la investigación en este campo más que ninguna otra publicación.

Antes del artículo de la "cognición caliente", tanto los modelos teóricos sobre el cambio conceptual como los procedentes de investigadores en Educación se centraban en principalmente en tres áreas: 1) la influencia de factores cognitivos tales como el conocimiento previo de los estudiantes, sus preconcepciones o concepciones erróneas (misconceptions), 2) cambios evolutivos en las representaciones de los jóvenes aprendices y 3 ) el diseño de métodos instruccionales que promovieran el cambio. Con escasas excepciones, estos modelos no reconocían apenas el papel de los factores afectivos, situacionales y motivacionales que influyen y en algunos casos determinan si el cambio se produce o no.

El artículo de la cognición caliente condujo a nuevos modelos de cambio conceptual que destacaron el papel de la motivación como un factor determinante del cambio. El Modelo de Reconstrucción Cognitiva del Conocimiento (CRKM o en castellano, MRCC) (Dole y Sinatra, 1998) y el Modelo Cognitivo-Afectivo de Cambio Conceptual (CAMCC o en castellano, MCACC)(Gregoire, 2003) son dos ejemplos de la tendencia "cálida" en los modelos de cambio conceptual que Paul inspiró. Ambos modelos incluyen componentes afectivos fuertes, tales como motivación, creencias sobre la eficacia, afecto e intenciones. Más concretamente, Paul avanzó su propia visión del papel de la motivación en el cambio conceptual en un capítulo reciente publicado en el libro editado por Schnotz, Vosniadou y Carretero, New Perspectives on Conceptual Change (Pintrich, 1999).

La influencia de Paul en mi pensamiento e ideas fue la razón por la que le propuse que co-editara conmigo un libro que estaba planificando sobre cambio conceptual intencional. Yo acababa de regresar de un sabático en la Universidad de Toronto / Ontario Institute for Studies in Education (OISE) y estaba entusiasmada con los planteamientos de los investigadores del OISE sobre el aprendizaje intencional. Ralph Reynolds, director de mi departamento, colaborador y amigo, me sugirió que le pidiera a Paul que co-editara conmigo el libro. 
Me recuerdo pensando que Paul debía tener peticiones de este tipo con tanta frecuencia que era difícil que mereciera la pena intentarlo, pero siguiendo el consejo de Ralph, me acerqué a Paul durante la conferencia anual de AERA, un par de meses más tarde, le dí una breve descripción de la idea del libro y le pregunté si estaría interesado en el proyecto. Para mi sorpresa, algunas semanas más tarde me contestó diciéndome que estaría muy interesado en co-editar el libro. Me puse muy contenta, pero aún no tenía ni idea de la maravillosa experiencia que estaba a punto de tener.

Esperaba que un académico tan ocupado y productivo asumiera un segundo plano. Esperaba que me ayudara a asegurar la colaboración de algunos autores y que, ocasionalmente, me diera algo de feedback sobre los capítulos. Para mi deleite, asumió un rol completo de coeditor. Paul fue un motor de guía intelectual en el proyecto y juntos, colaboramos en constuir una definición del constructo "cambio conceptual intencional". Nuestra definición final de cambio conceptual intencional como "iniciación consciente y dirigida a una meta que implica la regulación de aspectos cognitivos, metacognitivos y motivacionales para lograr un cambio en el conocimiento" lleva su marca (Sinatra y Pintrich, 2003, p. 6). Concretamente, no es sorprendente que añadiera "dirigido a una meta" como una característica definitoria del cambio conceptual intencional.

Como en muchas otras áreas en las que investigó, Paul ha dejado su marca indeleble en el campo de cambio conceptual. Es improbable que la motivación vuelva a ser ignorada en los futuros modelos de cambio conceptual. Es igualmente improbable que los investigadores en cambio conceptual olviden la profunda influencia de Paul Pintrich en nuestro pensamiento. Paul hizo que lo cambiáramos. ¡Qué maravilloso legado para un investigador del cambio conceptual!

\section{Referencias}

Dole, J.A., \& Sinatra, G.M. (1998). Reconceptualizing change in the cognitive construction of knowledge. Educational Psychologist, 33, 109-128.

Gregoire, M. (2003). Is it a challenge or a threat? A dual-process model of teachers' cognition and appraisal processes during conceptual change. Educational Psychology Review, 15, 147-179. 
Pintrich, P.R. (1999). Motivational beliefs as resources for and constraints on conceptual change. In W. Schnotz, S. Vosniadou, \& M. Carretero (Eds.), New perspectives on conceptual change (pp. 33-50). Oxford, UK: Elsevier Science.

Pintrich, P.R., Marx, R.W., \& Boyle, R.A. (1993). Beyond cold conceptual change: The role of motivational beliefs and classroom contextual factors in the process of conceptual change, Review of Educational Research, 63(2), 167-199.

Sinatra, G.M. \& Pintrich, P.R. (2003). The role of intentions in conceptual change learning. In G.M. Sinatra \& P.R. Pintrich (Eds.) Intentional Conceptual Change (pp.429-441). Mahwah, NJ: Erlbaum.

Dr. Gale M. Sinatra es profesora de Psicología Educativa en la Universidad de Nevada, Las Vegas, USA. Sus intereses de investigación incluyen cambio conceptual, aprendizaje e intencionalidad, así como el aprendizaje de la lectura, comprensión y evaluación. Su reciente libro, co-editado con Paul R. Pintrich, Intentional Conceptual Change, fue publicado por Lawrence Erlbaum Associates, U.S.A. en 2003.

- Dirección postal: 4505 Maryland Parkway, P.O. Box 453003, University of Nevada, Las Vegas, Las Vegas, NV 89154-3003, U.S.A 


\title{
Cambio Conceptual y el aprendiz intencio- nal esbozado por Paul R. Pintrich
}

\author{
Margarita Limón Luque \\ Departmento de Psicología Básica, Universidad Autónoma de Madrid
}

\section{España}

margarita.limon@uam.es

\begin{abstract}
Resumen
Este breve artículo incluye el texto escrito y leído por la autora, como representante del grupo de especialistas en cambio conceptual de EARLI (European Association for Research on Learning and Instruction) en la décima conferencia de EARLI (Padova, 26 al 30 Agosto de 2003), durante la sesión de homenaje a Paul Pintrich que sustituyó a su conferencia invitada. La autora intenta ofrecer una breve panorámica de las principales contribuciones de Paul Pintrich a la Psicología y la Educación y concretamente, a la investigación sobre cambio conceptual.
\end{abstract}

Palabras clave: Cambio Conceptual/ Aprendizaje intencional/Creencias epistemológicas/ Autorregulación/Motivación/ Psicología Cognitiva/ Educación 


\section{Introducción}

Durante la última conferencia de EARLI que tuvo lugar en Padua del 26 al 30 de Agosto de 2003, Paul estaba invitado a dictar una de las conferencias invitadas. Para sustituir su conferencia, Monique Boekaerts (University of Leiden, The Netherlands), como presidente y moderadora de la sesión en la que Paul iba a dar su conferencia, fue la encargada de coordinar y organizar una sesión de homenaje. Como ex-coordinadora del grupo de especialistas en cambio conceptual en el que Paul estaba muy involucrado y representando a este grupo al que pertenezco desde el año 1991, fui la encargada de escribir y leer en dicha sesión un texto en el que intenté reflejar algunas de las principales contribuciones de Paul tanto a la Psicología y la Educación, en general, como a la investigación en cambio conceptual, en particular.

Creo que este comentario general encaja bien con el propósito de esta parte del monográfico y por ello, he decidido mantener el texto original con cambios mínimos: he añadido un título, editado el texto, incluído las correspondientes referencias y escrito algunos comentarios personales finales.

\section{Cambio Conceptual y el aprendiz intencional esbozado por Paul R. Pintrich}

"Como representante del grupo de especial interés en cambio conceptual de EARLI del que Paul formaba parte, mi papel en esta sesión será presentar una breve panorámica de sus principales contribuciones al estudio del cambio conceptual y al desarrollo del pensamiento epistemológico. Sin embargo, me gustaría destacar también algunas contribuciones más generales que hizo al ámbito del aprendizaje y la instrucción. Empezaré con estas aportaciones de carácter general, para pasar después a las más específicas.

Creo que no puede llevarse a cabo una comprensión completa de las contribuciones de un científico sin conocer y entender a la persona que está detrás, esto es, su mente, su personalidad, las metas de su vida y sus valores.

En este sentido, en primer lugar, es importante señalar que una de las grandes pasiones de Paul, -junto con las olas y el "body surfing", y montar en bicicleta- fue enseñar y tutorizar a sus estudiantes. Teniendo en cuenta esta pasión, no es extraño que estuviera tan interesado en saber más sobre cómo mejorar el aprendizaje y la motivación de sus estudiantes. Esta dedicación 
a sus estudiantes, cuánto le gustaba cuidarlos y ofrecerles su apoyo incondicional creo que es una primera gran contribución de Paul.

Pienso que puede afirmarse sin duda alguna, que fue un modelo a seguir. Estaba absolutamente implicado en su trabajo de investigación, pero por encima de ello, lo que más quería era ser un buen profesor. Siempre decía que el premio del que estaba más orgulloso era el que sus estudiantes de licenciatura le dieron por su docencia. Además, a menudo aplicaba sus resultados de investigación y sus puntos de vista teóricos sobre motivación, aprendizaje y enseñanza a su propia práctica docente, tratando de mejorarla.

En segundo lugar, a Paul le encantaba hacer planes. Me atrevería a decir que era "un fanático de hacer planes”, establecer metar y ¡lograrlas!, aunque como buen autorregulador era lo suficientemente flexible para modificar sus planes cuando era necesario. Para Paul todo tenía que ser planificado con antelación (a menudo, mucho tiempo antes) y necesitaba saber los detalles de todo lo que se iba a hacer. Cuando lo conocías en el plano personal y no sólo en el profesional, quedaba claro por qué estaba tan interesado en el aprendizaje autorregulado y por qué destacaba el papel que asignaba a la planificación en él. También era evidente que toda su investigación estaba perfectamente planeada y encajaba en un esquema coherente que estuvo desarrollando cuidadosamente a lo largo de su carrera.

Para mostrarles hasta qué punto la planificación estaba presente en su investigación, les contaré una pequeña anécdota. Hace tres meses $^{1}$ me dijo que, puesto que iba a cumplir 50 años este año necesitaba planificar su investigación ¡para los próximos diez años, al menos! También tenía pensado cuándo se iba a retirar y lo que pensaba hacer en ese momento.

La autorregulación estaba muy presente en su vida y en su investigación. Como buen planificador y auorregulador, amaba la sistematización, el orden y la claridad. Creo que estas características están muy presentes en muchas de sus publicaciones. Era un maestro en escribir excelentes revisiones teóricas en las que se las arreglaba para encajar perspectivas teóricas muy diferentes, para destacar preguntas y cuestiones claves para la investigación futura y en sacar a la luz nuevos temas que permitieran generar progreso en nuestro campo de trabajo. Podría citar muchos de sus artículos como ejemplo de esto, pero quizás el más paradigmático sea el artículo publicado en la Review of Educational Research, sobre pensamiento epistemológico escrito jun- 
to a Bárbara Hofer (Hofer y Pintrich, 1997). Este artículo recibió el premio de la American Educational Research Association al Mejor Artículo de Revisión.

Tanto la investigación en cambio conceptual y pensamiento epistemológico, así como la relativa a autorregulación y motivación se ha beneficiado enormemente de estos excelentes ejemplos de cómo se debe escribir un artículo de revisión teórica. Desde mi punto de vista, esta es una segunda contribución general que hizo (para un ejemplo reciente en el ámbito de motivación, véase Pintrich, 2003).

En tercer lugar, y muy relacionado con su epistemología personal y su comprensión de la Psicología como disciplina, Paul se consideraba a sí mismo un científico. Como psicólogo que era, estaba interesado en saber más sobre los procesos cognitivos y motivacionales en sí mismos y también, pero no sólo, en las implicaciones que estos procesos tienen para la Educación.

En su último artículo como editor de Educational Psychologist (Pintrich, 2000a), destacó la tensión en nuestra disciplina entre las dimensiones básicas y aplicadas de la investigación. Esta tensión está vinculada también al uso de enfoques metodológicos cualitativos y cuantitativos y a la controversia entre la necesidad de realizar investigación en entornos reales o en situaciones de mayor control experimental para las que suele ser necesario el uso de laboratorios. Afirmaba que estas tensiones conducen a fragmentar el campo y a generar preguntas y cuestiones relativas a nuestra identidad como disciplina.

Paul propuso que buena parte de la investigación en psicología educativa debía guiarse tanto por metas que buscaran la comprensión científica en sí misma, como por metas que consideren prioritario generar un conocimiento científico útil. Creía que no toda nuestra investigación debería estar guiada sólo por esas últimas:

\footnotetext{
"los psicólogos educativos podrían unirse a otros psicólogos cognitivos, evolutivos, sociales, estudiosos de la personalidad, a la hora de desarrollar teorías y modelos que pudieran ser comprobadas de manera científica para incrementar nuestra comprensión básica de procesos como el aprendizaje, el desarrollo, la cognición y la motivación. Esta puede constituir una investigación experimental básica que tiene valor puesto que permite aumentar
}

\footnotetext{
${ }^{1}$ La autora se refiere a finales del mes de Abril de 2003.
} 
nuestra comprensión científica, incluso si en ese momento dicho conocimiento tiene pocas aplicaciones potenciales.

Al mismo tiempo, sin embargo, deberíamos involucrarnos en investigación que persiga tanto metas de comprensión científica como de utilidad y que permita comprender la actuación del individuo en contexto y el desarrollo de aplicaciones útiles para la educación." (Pintrich, 2000a; p. 224)

Este interés en destacar la necesidad, por un lado, de una mayor clarificación y por otro, de lograr acuerdo en las metas y metodología de nuestra disciplina como ciencia, junto con su llamada a la realización de investigación básica y aplicada, desarrollada tanto en contextos reales como de laboratorio, es otra de sus contribuciones generales.

En cuarto lugar, me gustaría destacar algunos de sus valores y de sus creencias sobre cómo deberían trabajar los científicos. Paul creía sinceramente en el aprendizaje colaborativo e intentaba ponerlo en práctica. Estaba siempre dispuesto a facilitar y generar contactos entre la comunidad científica en ambos lados del Atlántico. El SIG de cambio conceptual y otros miemros de EARLI nos hemos beneficiado ampliamente de ello. En los últimos años hemos organizado varias sesiones sobre cambio conceptual y pensamiento epistemológico tanto en los congresos de AERA como de EARLI que han contribuído ampliamente a estrechar los vínculos personales y profesionales entre los especialistas de nuestro campo. Estas actividades han facilitado y promovido la aparición de publicaciones conjuntas. A pesar de que Paul era uno de los especialistas más relevantes de nuestra área, era una persona muy sencilla, humilde, siempre accesible y dispuesto a apoyar y a ayudar a otros colegas. Lo recuerdo atendiendo muy a menudo, a una larga fila de personas que esperaban para hablar con él después de las sesiones y que atendía siempre con amabilidad y paciencia infinitas, escuchando a todo el mundo con una sonrisa, siempre disponible.

Me referiré a continuación a sus contribuciones específicas al ámbito del cambio conceptual y el pensamiento epistemológico. De manera general, creo que puede decirse que fue un pionero en tratar de unir los procesos motivacionales y cognitivos.

¿Cómo conectaba las creencias motivacionales y el aprendizaje autorregulado al cambio conceptual? La primera respuesta a esta excitante pregunta que en mi opinión, Paul fue refinando y completando más tarde en posteriores publicaciones, (véase, por ejemplo, Sinatra y Pintrich, 
2003) aparece en su artículo de 1993 junto a Ron Marx y Robert Boyle publicado en la Review of Educational Research bajo el título: "Beyond Cold Conceptual Change: the role of Motivational Beliefs and Classroom Contextual Factors in the Process of Conceptual Change" [Más allá del cambio conceptual frio: el papel de las creencias motivacionales y de los factores contextuales de la clase en el proceso de cambio conceptual].

Este artículo fue un punto de inflexión en el ámbito del cambio conceptual. Paul siempre decía que su implicación en EARLI se debió a este artículo, que si no lo hubiera escrito nunca lo habríamos invitado a nuestras reuniones del SIG. Y efectivamente fue así. Sólo unos meses después de que este artículo se publicara fue invitado a participar en el primer seminario del SIG de cambio conceptual de EARLI celebrado en Jena en Agosto de 1994, organizado por Wolfgang Schnotz y Stella Vosniadou. La reunión fue un éxito y Paul decidió hacerse miembro de EARLI y como él decía, desde entonces, ¡ya no se perdió ninguna conferencia de nuestra asociación! Decía que las conferencias de EARLI tenían muy buen nivel y contribuyó mucho gracias al gran apoyo que dió siempre a nuestra asociación, a atraer a otros colegas de Estados Unidos a participar en ellas, lo que debemos agradecerle mucho.

Más allá de esta anécdota sobre cómo su artículo de 1993 fue esencial poder contar con su participación en EARLI y concretamente en el SIG de cambio conceptual, explicaré con más detalle los vínculos y conexiones que empezó a establecer entre las piezas del "puzzle del aprendizaje y la instrucción": creencias motivacionales, autorregulación, conocimiento previo, contexto de la clase, y más tarde, la epistemología personal.

La crítica básica que él y sus colegas hicieron a los modelos de cambio conceptual desarrollados hasta entonces fue que no servían para describir adecuadamente el aprendizaje en contextos escolares. Las creencias motivacionales y las metas tienen un papel relevante en él, contribuyendo -o no - a que los aprendices logren activar el conocimiento conceptual adecuado.

Constructos motivacionales como la orientación a metas, el valor de la tarea, las creencias de eficacia y control actúan como mediadores en el proceso de cambio conceptual e influyen en factores cognitivos tales como la atención selectiva, activación del conocimiento previo, uso de un procesamiento más profundo o más superficial, identificación y solución de problemas, evaluación metacognitiva y control y regulación de la volición. 
También destacaban que los aprendices tienen intenciones, metas, propósitos y creencias que impulsan y sostienen su pensamiento. Y que estas creencias motivacionales influyen en cómo piensan los estudiantes cuando intentan adaptarse a las demandas y restricciones impuestas por el contexto de la clase.

El conocimiento previo constituye un marco que permite juzgar la validez de la nueva información que debe aprenderse. Pero este conocimiento previo puede estar también influído por lo que Hofer y Pintrich (2002) han llamado "epistemología personal" que corresponde a las creencias de los individuos sobre el conocimiento y su naturaleza (Pintrich, 2002, p. 390). Esto es, incluye las cogniciones y creencias sobre la certeza del conocimiento, su grado de simplicidad o complejidad, las fuentes de conocimiento o cómo éste se justifica.

Las creencias epistemológicas están también relacionadas con algunos aspectos motivacionales. Hofer y Pintrich (1997) sugieren que pueden funcionar como teorías implícitas que pueden dar lugar a ciertos tipos de metas de aprendizaje. Estas metas podrían realizar un papel de guías de la cognición y el comportamiento autorregulado. Por tanto, la epistemología personal se convirtió en otra pieza más que necesita ser ajustada en el puzzle.

Desde mi punto de vista, Paul ha contribuído definitivamente a dibujar y desarrollar una visión más compleja y completa del aprendiz, en la que los procesos cognitivos y motivacionales interactúan entre sí y además con el contexto en el que el proceso de aprendizaje tiene lugar.

Para dar una idea más precisa de cómo es este nuevo aprendiz, utilizaré una metáfora que elaboré en mi memoria de oposición a profesora titular (Limón, 2001) y que tuve oportunidad de discutir con Paul en varias ocasiones. En mi opinión, cabe destacar cuatro aprendices constructivistas metafóricos que ilustran el progreso hecho en nuestra área de trabajo desde los años 70. Estos cuatro aprendices son: el aprendiz lógico, el aprendiz especialista, el aprendiz situado y el aprendiz intencional.

El aprendiz lógico puede describirse como el aprendiz piagetiano por excelencia. Aprende a través de los mecanismos de asimilación y acomodación descritos por Piaget. La meta principal que este aprendiz debería lograr sería la construcción de las estructuras cognitivas adecuadas que se irían desarrollando con la edad. El cambio cognitivo se conseguiría como consecuencia de un proceso de re-equilibración iniciado por un conflicto cognitivo. 
El aprendiz especialista se describiría como el resultado de la adquisición de pericia en un determinado dominio. La meta de este aprendiz sería convertirse en un experto en algo. El aprendizaje se consideraría un proceso bastante específico de dominio, y por tanto, para explicar cómo se aprenden diferentes dominios sería necesario desarrollar modelos específicos. El cambio conceptual implicaría la reestructuración del conocimiento específico de dominio, lo que supondría cambios no sólo cuantitativos, sino también cualitativos.

El aprendiz situado se caracterízaría como un aprendiz social que pertenece a diferentes comunidades de práctica. Sería el producto de la perspectiva socioconstructivista del aprendizaje. Aprende en contexto y aprende con otros y de otros. El aprendiz situado construye conocimiento situado. El cambio conceptual no implicaría la reestructuración o sustitución del conocimiento previo por el conocimiento científico "correcto" o por el conocimiento disciplinar, sino la identificación de nuevos contextos en los que el conocimiento puede aplicarse para aprender de este modo, los posibles significados que puede tener.

Finalmente, el aprendiz intencional puede describirse como un aprendiz autorregulado. Un aprendiz que intenta y que quiere aprender algo, establece una meta y desarrolla un plan para lograrla. Este aprendiz intencional monitoriza y autorregula su motivación, su comportamiento y algunas de las características del contexto (Pintrich, 2000b), incluyendo los aspectos sociales del aprendizaje.

Los aprendices intencionales pueden fijarse como una posible meta de aprendizaje la modificación de su conocimiento. En este caso tiene lugar un proceso de cambio conceptual intencional. Gale Sinatra y Paul han editado recientemente un libro bajo este titulo "Intentional Conceptual Change" (Sinatra \& Pintrich, 2003), en el que se explora el papel de las intenciones en el cambio del conocimiento. Si bien es cierto que el constructo cambio conceptual intencional necesita ser explorado más ampliamente tanto en el plano teórico como empírico, ha permitido introducir el papel de las intenciones, las metas, la metacognición y la autorregulación en la discusión sobre el cambio conceptual, elaborando una visión mucho más compleja y completa de este proceso que la que lo reducía a la reestructuración del conocimiento previo.

Creo que la contribución de Paul en la construcción e investigación de este aprendiz intencional ha sido esencial y definitiva. Paul pensaba que los aspectos sociales y contextuales aún tenían que integrarse mucho mejor en el futuro en este aprendiz intencional. Este es otro de 
los retos que nos ha dejado abiertos. Deja muchos nuevos caminos y pistas para el desarrollo de investigación futura que permitirán continuar el trabajo en la dirección que él ha marcado, y a la que por desgracia, él ya no podrá seguir contribuyendo.

Ha sido un gran privilegio tenerlo en nuestro SIG, beneficiarnos de sus aportaciones intelectuales y personales y sobre todo, disfrutar de su amistad".

Hasta aquí el texto correspondiente a mi participación en la sesión de homenaje de la Conferencia de EARLI de Agosto de 2003. No quiero finalizar estas páginas sin añadir mi eterno agradecimiento a Paul por haber confiado tanto en mi, por poder contar siempre su apoyo cariñoso e incondicional durante todos estos años en los que hemos estado colaborando (cuando más lo necesité siempre estuvo ahí, sin fallar nunca), por su genial sentido del humor del que disfrutaba tanto, por darme la oportunidad de aprender tantas cosas de él a todos los niveles y por permitirme compartir con él tantos buenos ratos y muchas conversaciones estimulantes sobre Psicología y Educación. Empleando la terminología de nuestro campo de trabajo, él ha sido para mí un "dato anómalo", en este caso capaz por sí sólo de provocar en mí un cambio conceptual radical. "Muchas gracias por todo". Estas fueron mis últimas palabras cuando me despedí de él en el aeropuerto de Santiago de Chile, un mes antes de que muriera y con las que quiero terminar este pequeño homenaje en su memoria.

\section{Referencias}

Hofer, B.K., \& Pintrich, P.R. (1997). The development of epistemological theories : beliefs about knowledge and knowing and their relation to learning. Review of Educational Research, 67 (1), 88-140.

Hofer, B.K., \& Pintrich, P.R. (2002). Personal Epistemology. The psychology of beliefs about knowledge and knowing. Mahwah, NJ: Erlbaum.

Limón, M. (2001). Memoria de oposición. Madrid: Universidad Autónoma.

Pintrich, P.R. (2000a). Educational Psychology at the Millenium: A look back and a look forward. Educational Psychologist, 35 (4), 221-226.

Pintrich, P.R. (2000b). The role of goal orientation in self-regulated learning. In M. Boekaerts, P.R. Pintrich, \& M. Zeidner (Eds.), Handbook of Self-regulation (pp. 452-502). San Diego : Academic Press.

Pintrich, P.R. (2002). Conclusion: Future challenges and directions for theory and research on 
personal epistemology. In B.K. Hofer \& P.R. Pintrich (Eds.), Personal Epistemology. The psychology of beliefs about knowledge and knowing (pp. 389-414). Mahwah, NJ: Erlbaum.

Pintrich, P.R. (2003). A motivational science perspective on the role of student motivation in learning and teaching contexts. Journal of Educational Psychology, 95(4), 667-686.

Pintrich, P.R.; Marx, R.W. \& Boyle, R.A. (1993). Beyond cold conceptual change: the role of motivational beliefs and classroom contextual factors in the process of conceptual change. Review of Educational Research, vol. 63 (2), 167-200.

Sinatra, G.M. \& Pintrich, P.R. (Eds.)(2003). Intentional Conceptual Change. Mahwah, NJ: Lawrence Erlbaum Associates.

Margarita Limón es profesora titular de la Facultad de Psicología de la Universidad Autónoma de Madrid (España). Su investigación se ha centrado en razonamiento en dominios específicos de conocimiento, razonamiento informal, cambio conceptual, creencias epistemológicas y más recientemente, está traajando en aprendizaje con materiales multimedia. Junto con Lucia Mason, ha sido coordinadora del SIG de cambio conceptual de EARLI (European Association for Research on Learning and Instruction), desde 1997 a 2001.

Paul y ella han participado y organizado numerosas sesiones sobre cambio conceptual y sobre creencias epistemológicas desde 1994, tanto en las conferencias anuales de EARLI y AERA como en las reuniones de los SIGs de estas asociaciones. Ha colaborado también con Paul en la elaboración de dos libros recientes sobre cambio conceptual: Limón, M. \& Mason, L.(Eds.)(2002), Reconsidering Conceptual Change: Issues in Theory and Practice. Dordretch, The Netherlands: Kluwer Academic Press y Sinatra, G.M. \& Pintrich, P.R. (Eds.)(2003). Intentional Conceptual Change. Mahwah, NJ: Lawrence Erlbaum Associates.

Estaba empezando a desarrollar con Paul algunos proyectos de investigación y docencia en Sudamérica. Con este motivo, ambos organizaron en Junio de 2003 un seminario bajo el título Current Issues in Learning and Instruction que se celebró en Santiago de Chile (Chile) apenas un mes antes de que Paul falleciera. Habían planeado la traducción al español del libro de Paul y Dale H. Schunk, Motivation in Education, $2^{\text {nd }}$ edition, que se publicará en 2004 y estaban trabajando juntos en nuevas publicaciones.

\section{Dirección postal:}

Margarita Limón. Dpto. de Psicología Básica. Facultad de Psicología. Universidad Autónoma de Madrid. Cantoblanco, 28049 Madrid (Spain). 


\title{
Identificando retos en la investigación sobre el aprendizaje autorregulado: contribuciones de Paul R. Pintrich
}

\author{
Philip Winne
}

Self-Regulated Learning and Learning Technologies, Faculty of Education Simon Fraser University, Burnaby

\section{Canadá}

winne@sfu.ca

\section{Resumen}

El profesor Winne subraya en su comentario tres maneras en las que Paul R. Pintrinch contribuyó al desarrollo del aprendizaje autorregulado: asumiendo un papel de director benelovente y guía del trabajo comunitario, como investigador empírico y como un teórico constructivo e inspirador siempre atento e interesado en las implicaciones para la práctica educativa. Winne ilustra estas tres facetas del trabajo de Paul en esta área.

Palabras clave: Aprendizaje autorregulado / Aprendizaje intencional/ Psicología Cognitival Motivación / Metas. 
Durante las últimas dos décadas la teoría y la investigación sobre el aprendizaje autorregulado (SRL en inglés, en castellano, AAR) ha construído sus cimientos al amparo de la revolución cognitiva en Psicología. Concretamente, la teoría del aprendizaje autorregulado elabora la influyente unidad prueba-operación-prueba propuesta por Miller, Galanter y Pribram (1960) de un modo que no sólo preserva, sino que acentúa la humanidad de los aprendices. Lo hace a través de un simple, pero profundo axioma: los aprendices son agentes. Agentes que eligen y toman decisiones sobre su comportamiento. Sus elecciones están basadas, pero no determinadas por los ambientes en los que habitan. Quizás de modo más específico y significativo, puede decirse que los agentes y los entornos en los que se mueven interacciones en el tiempo de tal modo que cada uno da forma al desarrollo del otro. Debido a que la teoría del aprendizaje autorregulado adopta una perspectiva del aprendizaje que es inherentemente dinámica y a la vez impredecible, si no aleatoria, presenta desafíos especiales a aquellos que pretenden comprender sus elementos, su estructura y el modo en el que el aprendizaje autorregulado da cuenta del comportamiento humano y el aprendizaje.

No es una exageración decir que Paul R. Pintrich estaba entre los académicos del planeta más influyentes, reflexivos y colaboradores que han trabajado para hacer avanzar la teoría y la investigación sobre el aprendizaje autorregulado. ¿Qué evidencias hay para sostener esta afirmación rotunda y atrevida? Citaré sólo tres breves ejemplos de entre los muchos que podrían enumerarse.

Junto a sus cercanos colegas Monique Boekaerts de la Universidad de Leiden (Holanda) y Moshe Zeidner de la Universidad de Haifa (Israel), Paul fue el arquitecto del Handbook of Self-Regulation (2000). Esta colección enciclopédica de trabajo sobre el aprendizaje autorregulado permitió presentar por primera vez una visión panorámica de las teorías sobre qué es el aprendizaje autorregulado, cómo se ha investigado y qué efectos se han asociado con él. El papel de Paul como facilitador de este trabajo de colaboración creando este recurso único, es sólo un ejemplo de su dedicación a un enfoque basado en el trabajo en equipo, para lograr hacer avanzar el trabajo realizado en este área y para cultivar el desarrollo de recursos que ayudaran a otros a hacer avanzar la ciencia del aprendizaje autorregulado.

Trabajos empíricos como los que pueblan los capítulos del Handbook que Paul coeditó proporcionan los medios adecuados para validar la teoría del aprendizaje autorregulado. 
La ciencia del aprendizaje autorregulado se quedaría encallada sin investigación empírica meticulosamente diseñada, ejecutada con cuidado y presentada en lúcidos informes. Paul fue un prolífico e inspirador investigador empírico cuyo trabajo fijó las bases de este campo de investigación. Un ejemplo reciente de esto es su estudio (2000) en el que demuestra que las metas orientadas al resultado no necesariamente orientan a los aprendices a trabajar de modo que minen su adaptación o a forzarlos hacia caminos que los condenan al fracaso. Una característica especialmente ventajosa de este estudio fue la demostración de la significación longitudinal de la orientación a metas con respecto a las expresiones del aprendizaje autorregulado, tales como el "auto-lesionamiento" (self-handicapping) y el correr riesgos. Sobre todo, el corpus de trabajos empíricos de Paul construyó puentes clave que permitieron unir los aspectos cognitivos y motivacionales del aprendizaje autorregulado, iluminando más que complicando el conocimiento de este ámbito de trabajo.

Los teóricos deben absorber y en ocasiones luchar con trabajo empírico como el producido por Paul y otros. Confrontándolo continuamente e incluso "luchando a brazo partido" con los desafíos que supone para la teoría, nosotros, los que trabajamos en el campo del aprendizaje autorregulado, como los que lo hacen en otras áreas científicas, desarrollamos una empresa autorreguladora. Puesto que el aprendizaje autorregulado implica una esfera de acción tan amplia del aprendizaje y cambio humanos, la síntesis, construcción crítica, la reunión de datos y la reinterpretación de resultados son especialmente desafiantes. Es en esta arena en la que Paul hizo enormes y frecuentes contribuciones a la ciencia del aprendizaje autorregulado. Una de las últimas publicaciones de Paul (2003) sobre motivación ilustra su excelente capacidad para hacer esto. En este artículo, Paul aborda los aspectos centrales de la ciencia de la motivación tocando a menudo, temas centrales del aprendizaje autorregulado. En sólo unas dieciséis páginas ofrece no sólo una sólida síntesis de temas y fundamentadas sugerencias para la práctica educativa, sino que también presenta inspiradoras directrices para el avance del campo en el futuro. Tanto esta como otras de sus numerosas síntesis de investigación permanecerán como modelos para ser estudiados en el futuro, que ofrecen además, herramientas conceptuales para avanzar en la ciencia del aprendizaje autorregulado. A través de estas publicaciones, las habilidades académicas de Paul y sus insights guiarán tanto la ciencia como la práctica educativa en los años venideros.

De estos tres modos, asumiendo un papel de director benelovente y guía del trabajo comunitario, como investigador empírico y como un teórico constructivo e inspirador siempre 
atento e interesado en las implicaciones para la práctica educativa, el legado de Paul al campo del aprendizaje autorregulado es distintivo, sustancial y pionero. Yo - y estoy seguro de predecir con toda seguridad la opinión de los colegas que trabajaron con él- tendremos que esforzarnos mucho para igualar los estándares que Paul alcanzó y fijó. Es una tarea que asumiremos en homenaje a nuestro más querido compañero.

\section{Referencias}

Boekaerts, M., Pintrich, P. \& Zeidner, M. (Eds.), Handbook of self-regulation. Orlando, FL: Academic Press.

Pintrich, P.R. (2000). Multiple goals, multiple pathways: The role of goal orientation in learning and achievement. Journal of Educational Psychology, 92, 544-555.

Pintrich, P.R. (2003). A motivational science perspective on the role of student motivation in learning and teaching contexts. Journal of Educational Psychology, 94, 667-686.

Philip Winne es profesor y ocupa la Canada Research Chair en aprendizaje autorregulado y en aprendizaje de teconologías en la Facultad de Educación de la Universidad Simón Fraser. Actualmente es co-editor de Educational Psychologist y editor asociado del British Journal of Educational Psychology. Winne es tamién Fellow de la asociaciones de Psicología americana y canadiense. Sucedió a Paul en la presidencia de la División 15 (Educational Psychology) de la APA: Aunque nunca colaboró directamente con Paul en investigación, sien embargo, a menudo disfrutaban de discusiones profundas y llenas de energía sobre el aprendizaje autorregulado y sobre el campo de la Psicología Educativa, en general.

\section{Dirección postal:}

Philip Winne. Faculty of Education. Simon Fraser University. Burnaby, BC V5A 1S6

Canada. 


\title{
Sobre la obra de Paul R. Pintrich: La autorregulación de los procesos cognitivos y motivacionales en el contexto educativo
}

\author{
Ignacio Montero y María José de Dios \\ Departamento de Psicología Social y Metodología, Universidad Autónoma de \\ Madrid, Madrid
}

\section{España}

nacho.montero@,uam.es

\section{Resumen}

El objetivo de este artículo es presentar y comentar la obra de P.R. Pintrich en la parte ligada al modelo teórico que desarrolló sobre la autorregulación del aprendizaje y la motivación en contextos académicos. Partiendo de numerosos estudios empíricos fue elaborando a lo largo de más de dos décadas de trabajo un modelo teórico en la que los elementos motivacionales y cognitivos se encuentran en estrecha conexión, teniendo siempre en cuenta el contexto en el que tiene lugar el aprendizaje. Desde su perspectiva, tanto los procesos motivacionales como los cognitivos pueden ser regulados por el propio aprendiz.

Palabras clave: Autorregulación / Motivación / Aprendizaje 


\section{Introducción}

Desde el inicio de su carrera como investigador, Pintrich otorga una gran importancia al contexto de aprendizaje y a los factores sociales que influyen en el aula. Puede decirse que su principal contribución en el área que nos ocupa ha sido proporcionar tanto propuestas teóricas integradoras de los factores cognitivos, motivacionales y contextuales como datos empíricos abundantes que demuestran las estrechas conexiones entre dichos factores.

En este estudio de la relación entre los elementos motivacionales, cognitivos y contextuales en el aprendizaje, su trabajo se ha centrado en investigar el uso de estrategias y de los procesos de autorregulación, en la esfera de lo cognitivo, y paralelamente, en el ámbito de lo motivacional, en el papel de las metas y de la orientación de los alumnos hacia uno u otro tipo en el proceso de aprendizaje (Pintrich, 2000a).

\section{La motivación, la cognición y el contexto de aprendizaje en la teoría de Pintrich}

A la hora de explicar los procesos de aprendizaje en contextos académicos, Pintrich (1994; 2000b, 2003 a) destaca tres componentes:

a) Motivacionales: la orientación a metas de logro, las expectativas de éxito y fracaso, las autopercepciones de competencia y habilidad (creencias de autoeficacia), creencias de control, el valor asignado a la tarea y las reacciones afectivas y emocionales.

b) Cognitivos: las estrategias de autorregulación cognitiva, las estrategias aprendizaje, la metacognición, la activación de conocimiento previo, etc.

c) Relativos al contexto de aprendizaje: las características de la tarea, el contexto en el que tiene lugar la actividad, la percepción del alumno de ambos aspectos, las metas que se proponen en el aula, la estructura de trabajo en la clase, los métodos de enseñanza, la conducta del profesor y el tipo de interacciones que se establece entre alumnos y entre profesores y alumnos.

Pintrich considera que existe una relación bidireccional entre estos tres tipos de componentes que, además se encuentran estrechamente unidos (García y Pintrich, 1994, Pintrich, 2000b). En los apartados siguientes, explicaremos con algo más de detalle estas relaciones. 


\section{Los factores motivacionales en la teoría de Pintrich}

Pintrich concibe la motivación escolar como un constructo con múltiples elementos o factores (Linnenbrink y Pintrich, 2002b). De entre todos los factores motivacionales (para una revisión detallada, puede consultarse Pintrich, 2003a, Pintrich, 2002) vamos a destacar aquí, por ser dos de los elementos más destacados en su obra, el papel de las metas y de la orientación a metas de los aprendices y las expectativas de autoeficacia.

Pintrich (2000a) plantea una taxonomía con cuatro posibles orientaciones a metas: aproximación al aprendizaje, evitación del aprendizaje, aproximación al resultado y evitación del resultado (Linnenbrink y Pintrich, 2000). Estas cuatro posibilidades resultan de combinar dos dimensiones: tipo de orientación a meta (orientación al proceso de aprendizaje versus orientación al resultado) y aproximación o evitación hacia ellas (Pintrich, 2002).

Esta clasificación amplía la tradicional de la teoría normativa que distinguía entre metas de aprendizaje y de resultado, y sólo planteaba el estudio de la aproximación y evitación dentro de la orientación a metas de resultado. Cada una de estas cuatro posibles orientaciones conlleva relaciones distintas con otros elementos del aprendizaje autorregulado (Linnenbrink y Pintrich, 2000; Pintrich, 2000b).

Suponen también que los aprendices se impliquen y afronten las tareas de aprendizaje de diferente manera. Para validar su teoría, Pintrich ha estudiado la orientación a metas de los alumnos en diversos contextos y disciplinas (Wolters, Yu y Pintrich, 1996, Pintrich y Zusho, 2001). Además, ha analizado las diferencias entre las metas que persiguen los alumnos y su percepción de las metas escolares (Linnenbrink y Pintrich, 2002a), encontrando una relación recíproca entre ambos aspectos.

Desde sus primeros trabajos (por ejemplo, (Blumenfeld, Pintrich, Meece y Wessels, 1982, Pintrich y Blumenfeld, 1985, Blumenfeld, Pintrich y Hamilton, 1986) Pintrich estudió las expectativas de autoeficacia, elemento que consideró clave para que los alumnos se comprometan con la tarea y logren aprender (Linnenbrink y Pintrich, 2003). Las creencias de autoeficacia influyen no sólo en la motivación hacia la tarea, sino también en el comportamiento y los procesos cognitivos que se ponen en funcionamiento durante la realización de la actividad de aprendizaje. 
Los resultados obtenidos por Pintrich muestran que estos componentes motivacionales son más o menos relevantes (e incluso pueden desempeñar funciones diferentes) a lo largo del proceso de aprendizaje. Por ejemplo, Pintrich y de Groot, 1990, encontraron que el valor intrínseco que se otorga a la actividad influye especialmente en la implicación inicial del sujeto en la tarea. Es también al comienzo de la actividad cuando el sujeto adopta una orientación a meta determinada (Pintrich, 2000b), que se va adaptando durante la ejecución y puede modificarse, por tanto, a lo largo de la misma.

En cambio, otros elementos, como las creencias de autoeficacia, tienen un papel más relevante en fases posteriores del proceso de ejecución de la tarea. Las reacciones emocionales suelen producirse cuando los aprendices han completado la actividad de aprendizaje, como consecuencia de las explicaciones que los aprendices generan para explicar su éxito o su fracaso (atribuciones causales).

Estas atribuciones pueden modificar la percepción de la autoeficacia y las expectativas de éxito, así como el valor otorgado a la tarea (Pintrich y Schunk, 2002). Parece claro, por tanto, que existe una significativa interacción entre los diferentes elementos motivacionales a lo largo de todo el proceso de aprendizaje que se traduce en que algunos de ellos pueden modificar otros antes, durante y al final de la actividad.

\section{La relación entre elementos motivacionales y cognitivos}

Según Pintrich (2003b), la integración de los elementos motivacionales y cognitivos es necesaria para tener una visión completa del proceso de aprendizaje en el contexto escolar, así como para comprender las dificultades que aparecen en el proceso de instrucción.

Muchos de sus trabajos (Pintrich y de Groot, 1990, Pintrich, Roeser y de Groot, 1994, Pintrich y Zusho, 2002) han relacionado los factores motivacionales con elementos cognitivos, especialmente con el uso de estrategias de autorregulación (para una revisión, ver Pintrich, 2000b). Estos trabajos han demostrado que los procesos motivacionales pueden facilitar o dificultar el desarrollo de la autorregulación (Pintrich y Zusho, 2002).

Por ejemplo, sujetos con determinadas orientaciones a metas -concretamente con orientación a metas de aprendizaje- suelen realizar más intentos de controlar su propia cogni- 
ción y utilizan con más frecuencia determinadas estrategias de aprendizaje (Pintrich y de Groot, 1990, Pintrich et al, 1994).

Además, la percepción de autoeficacia que tiene el alumno y el valor intrínseco que otorga a la actividad se relacionan con la implicación cognitiva en la tarea y con el rendimiento en la misma (Pintrich y de Groot, 1990). Los factores motivacionales y cognitivos se relacionan de forma tan estrecha que se puede llegar a distinguir distintos perfiles conjuntos de motivación y cognición en los alumnos (Pintrich y García, 1993).

\section{La relación entre procesos motivacionales, cognitivos y el contexto de aprendizaje}

Para Pintrich, el contexto es un factor esencial en el aprendizaje. Pintrich (1994), resalta la relación entre las creencias motivacionales y la influencia que determinadas características del contexto educativo pueden tener sobre la motivación de los alumnos. (Linnenbrink y Pintrich, 2001, 2003).

Su visión, a diferencia de la que se defiende en otras teorías (ver Bandura, 1997; Csikszentmihalyi, 1988; Deci y Ryan, 1985; Dweck y Elliott, 1983), refleja que la motivación del estudiante va más allá del propio individuo ya que, aunque éste tiene un papel activo en la regulación de su motivación, se ve claramente influido por el contexto. Y este, a su vez, se modifica por el comportamiento del alumno. Con ello, Pintrich deja claro que cualquier intervención motivacional en el aprendizaje de los alumnos debe suponer una atención no sólo al individuo, sino también a lo que lo rodea.

De ahí, que nunca perdiera de vista la aplicación de su teoría a la práctica educativa. y concretamente, a las posibilidades de crear un contexto lo más propicio posible para favorecer el aprendizaje con especial énfasis en el papel del docente en esta labor. Al mismo tiempo, insiste en la necesidad de enseñar a los aprendices a autorregularse (Hofer, Yu y Pintrich, 1998, Pintrich, 2000b).

\section{Referencias}

Bandura A. (1997). Self-Efficacy: The exercise of control. New York: Freeman.

Blumenfeld, Ph. C., Pintrich, P. R., y Hamilton, V. L. (1986). Children's concepts of ability, 
effort, and conduct. American Educational Research Journal, 23(1), 95-104.

Blumenfeld, Ph. C., Pintrich, P. R., Meece, J., y Wessels, K. (1982). The formation and role of self perceptions of ability in elementary classrooms. Elementary School Journal, 82(5), 401-420.

Csikszentmihalyi, M. (1988). The flow experience and its significance for human psychology. En M. Csikszentmihalyi e I.S. Csikszentmihalyi (Eds.), Optimal experience: Psychological studies of flow in consciousness (pp.15-35). Cambridge, MA: Cambridge University Press.

Deci, E. L., y Ryan, R. M. (1985). Intrinsic motivation and self-determination in human behavior. New York: Plenum Press.

Dweck, C. S., y Elliott, E. S. (1983). Achievement motivation. En P.H. Minsen y E.M. Hetherington (Eds.), Handbook of child psychology, vol IV: Social and personality development (pp. 643-691). New York, NY: Wiley

García, T., y Pintrich, P. R. (1994). Regulating motivation and cognition in the classroom: The role of self-schemas and self-regulatory strategies. En D.H. Schunk y B.J. Zimmerman (Eds.), Self-regulation of learning and performance: Issues and educational applications (pp. 127-153). Hillsdale, NJ: Lawrence Erlbaum Associates.

Hofer, B. K., Yu, Sh. L., y Pintrich, P. R. (1998). Teaching college students to be selfregulated learners. En D.H. Schunk y B.J. Zimmerman (Eds). Self-regulated learning: From teaching to self-reflective practice (pp.57-85). New York, NY: Guilford Publications.

Linnenbrink, E. A., y Pintrich, P. R. (2000). Multiple pathways to learning and achievement: The role of goal orientation in fostering adaptive motivation, affect and cognition. En C. Sansone y J.M. Harackiewicz (Eds.), Intrinsic and extrinsic motivation: The search for optimal motivation and performance (pp.195-227). San Diego, CA: Academic Press.

Linnenbrink, E. A., y Pintrich, P. R. (2001). Multiple goals, multiple contexts: The dynamic interplay between personal goals and contextual goal stresses. En S. Volet, y S. Järvelä (Eds.), Motivation in learning contexts: Theoretical advances and methodological implications (pp. 251-269). Elmsford, NY: Pergamon Press.

Linnenbrink, E. A., y Pintrich, P. R. (2002a). Achievement goal theory and affect: An asymmetrical bidirectional model. Educational Psychologist, 37(2), 69-78.

Linnenbrink, E. A., y Pintrich, P. R. (2002b). Motivation as an enabler for academic success. School Psychology Review, 31(3), 313-327. 
Linnenbrink, E. A., y Pintrich, P. R. (2003). The role of self-efficacy beliefs in student engagement and learning in the classroom. Reading \& Writing Quarterly: Overcoming learning difficulties, 19(2), 119-137.

Pintrich, P. R. (1994). Student motivation in the college classroom. En K.W. Prichard y R.M. Sawyer (Eds.), Handbook of college teaching: Theory and applications (pp. 23-43). Westport, CT: Greenwood Press/Greenwood Publishing Group.

Pintrich, P. R. (2000a). Multiple Goals, Multiple Pathways: The role of Goal Orientation on Learning and Achievement. Journal of Educational Psychology, vol.92 (3), 544-555.

Pintrich, P. R. (2000b). The role of goal orientation in self-regulated learning. En M. Boekaerts, P.R. Pintrich, y M. Zeidner (Eds.), Handbook of self-regulation (pp. 452502). San Diego, CA: Academic Press.

Pintrich, P.R. (2002). The role of goals and goal orientation. En P.R. Pintrich y D. H. Schunk (Eds.), Motivation in education: Theory, research, and applications. $2^{\text {nd }}$. edition.(pp. 190-242). Englewood Cliffs, NJ: Prentice Hall. Trad. castellano, Motivación y educación: teoría, investigación y aplicaciones. Madrid: Pearson-Prentice Hall, 2004.

Pintrich, P. R. (2003a). A motivational science perspective on the role of student motivation in learning and teaching contexts. Journal of Educational Psychology, 95(4), 667-686.

Pintrich, P. R. (2003b). Motivation and classroom learning. En W.M. Reynolds y G.E. Miller (Eds). Handbook of Psychology: Educational Psychology, Vol. 7 (pp.103-122). New York, NY: John Wiley \& Sons.

Pintrich, P. R., y Blumenfeld, Ph, C. (1985). Classroom experience and children's selfperceptions of ability, effort, and conduct. Journal of Educational Psychology, 77(6), 646-657.

Pintrich, P. R., y de Groot, E. V. (1990). Motivational and self-regulated learning components of classroom academic performance. Journal of Educational Psychology, 82(1), 33-40.

Pintrich, P.R., y García, T. (1993). Intraindividual differences in students' motivation and selfregulated learning. Zeitschrift fuer Paedagogische Psychologie, 7(2-3), 99-107.

Pintrich, P. R., Roeser, R. W., y de Groot, E. V. (1994). Classroom and individual differences in early adolescents' motivation and self-regulated learning. Journal of Early Adolescence, 14(2), 139-161.

Pintrich, P. R., y Schunk, D. H. (2002). Motivation in education: Theory, research, and applications. $2^{\text {nd }}$. edition. Englewood Cliffs, NJ: Prentice Hall. Trad. castellano, Motivación y educación: teoría, investigación y aplicaciones. Madrid: Pearson-Prentice Hall, 2004. 
Pintrich P. R., y Zusho, A. (2001). Goal orientation and self-regulated learning in the college classroom: A cross-cultural comparison. En F. Salili y Ch. Chiu (Eds.), Student motivation: The culture and context of learning (pp. 149-169). Dordrecht, Netherlands: Kluwer Academic Publishers.

Pintrich P. R., y Zusho, A. (2002). The development of academic self-regulation: The role of cognitive and motivational factors. En A. Wigfield y J.S. Eccles (Eds.), Development of achievement motivation (pp. 249-284). San Diego, CA: Academic Press.

Weiner, B. (1986). An attributional theory of motivation and emotion. New York: SpringerVerlag.

Wolters, Ch. A., Yu, Sh. L., y Pintrich, P. R. (1996). The relation between goal orientation and students' motivational beliefs and self-regulated learning. Learning \& Individual Differences, 8(3), 211-238.

Ignacio Montero Garcia-Celay es profesor titular de Departamento de Psicología Social y Metodología de la Universidad Autónoma de Madrid (España). Su Tesis Doctoral y su invesigación se han centrado en la evaluación de la motivación en contextos escolares. Más recientemente, ha abordado el estudio de la función motivacional del habla privada.

Montero contactó con Paul Pintrich en algunas ocasiones en Conferencias de AERA y cuando Paul fue invitado a la Universidad Autónoma de Madrid para participar en el segundo seminario internacional sobre cambio conceptual (1998) y para impartir un seminario dentro de un curso de doctorado del programa “Aprendizaje e Instrucción” del Departamento de Psicología Básica (2000). Recientemente, ha colaborado en la traducción del libro de Paul y Dale H. Schunk, Motivation in Education [Motivación y Educación] que será publicado en castellano por Pearson-Merrill Prentice Hall en 2004.

María José de Dios es licenciada en Psicología y estudiante de doctorado de la Facultad de Psicología de la Universidad Autónoma de Madrid.

\section{Dirección para correspondencia:}

Ignacio Montero García-Celay. Dpto. Psicología Social y Metodología. Facultad de Psicología. Universidad Autónoma de Madrid. Cantoblanco, 28049, Madrid. 


\title{
El factor humano
}

\author{
Patricia A. Alexander
}

Department of Human Development, University of Maryland, College Park

USA

palexand@umd.edu

\section{Resumen}

A lo largo de su comentario, la autora destaca, además de las aportaciones académicas y profesionales, la extraordinaria capacidad de Paul Pintrich para actuar como mentor de jóvenes estudiantes, así como para alimentar y apoyar la carrera de otros colegas. Pintrich poseía en abundancia algunas características esenciales de un buen mentor: conocimiento y perspectiva del campo, respeto y admiración de la comunidad, gran habilidad para trabajar bien con otros y una verdadera pasión por la Psicología y la Educación.

Palabras clave: Mentor/Educación/ Psicología educativa/ Aprendizaje/ Enseñanza 
Cuando los miembros de una comunidad de investigación evalúan las contribuciones académicas de un colega, éstas se miden habitualmente en términos del número de publicaciones en revistas que acrediten proceso de revisión entre iguales, número de citas, contribuciones en volúmenes especialmente relevantes en el área, responsabilidades editoriales, cargos que impliquen liderazgo, premios recibidos y ponencias presentadas en congresos nacionales e internacionales. Teniendo en cuenta todas estas medidas cuantitativas, Paul R. Pintrich era un académico de primer orden. Por ejemplo, el papel de Paul en generar la agenda de investigación de la Psicología Educativa en áreas como creencias epistemológicas, cambio conceptual, motivación y autorregulación está bien documentado y acreditado en las contribuciones de otros autores de esta parte del monográfico que han precedido a la mia. Por tanto, dejo esta parte a mis estimados colegas para profundizar más en la relevancia de Paul a las esferas de la investigación.

Los logros en un campo como el de la Psicología Educativa no pueden medirse solamente en términos de pulicaciones y ponencias. Hay un factor humano que debe considerarse también. Para ser más precisa, los académicos de primer orden ponen sus fuerzas y energías en alimentar y cuidar la carrera de otros que esperan se conviertan en la siguiente generación de académicos, asegurando, de ese modo, la continuidad de la salud y prosperidad del dominio. Es debido a esta extraordinaria capacidad para actuar como mentor de jóvenes académicos, por lo que celebro especialmente las aportaciones de Paul R. Pintrich a la Psicología Educativa.

Sólo la lista de antiguos estudiantes de postgrado y doctorado de Paul es impresionante. Muchos de ellos están ya dejando sus huellas en el campo de la Psicología Educativa, entre ellos, Eric Anderman, Lynley Anderman, Teresa Garcia, Barbara Hofer, Elizabeth Linnenbrink, Timothy Urdan, and Christopher Wolters. A través de las asociaciones de estudiantes graduados dentro de la Asociación Americana de Investigación Educativa (AERA) y de la División 15 (Psicología Educativa) de la Asociación Americana de Psicología (APA), Paul fue capaz de influir en la vida profesional de estudiantes graduados procedentes de instituciones de todo Estados Unidos. Pero no fueron sólo los estudiantes graduados los que se beneficiaron de la sabiduría de Paul. Algunos de los investigadores que participan en esta parte del monográfico se han beneficiado también de su colaboración con Paul Pintrich, yo incluída. 
La habilidad para apoyar el desarrollo académico en todas estas etapas del desarrollo profesional están incluídas en algunas de las características imprescindibles de un buen mentor: conocimiento y perspectiva del campo, respeto y admiración de la comunidad, habilidad para trabajar bien con otros y verdadera pasión por el dominio. Paul poseía todas estas características en abundancia. Por ejemplo, los buenos mentores deben tener un extenso conocimiento del dominio en el que trabajan y la habilidad para comunicar este conocimiento a otros. Si se revisan sus publicaciones, queda muy claro que Paul tenía un punto de vista privilegiado y muy especial del campo de la Psicología Educativa y era capaz de sintetizar e integrar brillantemente, literatura compleja y amplia (véase, por ejemplo, Hofer \& Pintrich, 1997, Pintrich, 1994; 2003; Pintrich, Marx, \& Boyle, 1993; Pintrich \& Schunk, 2001).

En segundo lugar, un buen mentor debe suscitar un amplio respeto en la comunidad, así como el de aquellos que aprenden de él o ella. En este sentido, el liderazgo de Paul dentro de la Psicologia Educativa estaba firmemente establecido. Entre sus muchos puestos de liderazgo cabe destacar su papel como ex-presidente de la División 15 de APA o como ex-editor de Educational Psychologist y miembro de numerosos consejos de redacción y comités editoriales de revistas del campo.

Además, Paul demostró reiteradamente su habilidad para trabajar con otros, manteniendo siempre su alto nivel en su trabajo con todos los miembros de la comunidad de investigación. Esta habilidad para ofrecer su apoyo y su guía por un lado, y sus altas expectativas de erudición académica, por otro, no son fácilmente alcanzables. Sin embargo, Paul consiguió todo esto con elegancia y una persistente pasión por la búsqueda del conocimiento.

Por tanto, debemos recordar las contribuciones de Paul a la comunidad de investigación como mentor excepcional tanto como sus muchas aportaciones al campo de la Psicología Educativa o su erudición respecto a la literatura e investigación sobre creencias epistemológicas, cambio conceptual, motivación y autorregulación. Es a través de mentores sabios, respetados, capaces y apasionados como el futuro de las disciplinas, como es el caso de la Psicología Educativa, queda asegurado. No cabe duda de que Paul R. Pintrich poseía estas cualidades en un nivel excepcional. Fue un mentor con un conocimiento sin igual, muy respetado en el campo y apasionado respecto a él. Sus contribuciones a la Psicología Educativa cambiarán y mejorarán para siempre nuestra disciplina gracias a sus aportaciones a los miembros de la comunidad. El factor humano me ha tocado a mí y a otros muchos. 


\section{References}

Hofer, B.K., \& Pintrich, P.R. (1997). The development of epistemological theories: Beliefs about knowledge and knowing and their relation to learning. Review of Educational Research, 67, 88-140.

Pintrich, P.R. (1994). Continuities and discontinuities: Future directions for research in Educational Psychology. Educational Psychologist, 29, 137-148.

Pintrich, P.R. (2000). An achievement goal theory perspective on issues in motivation terminology, theory, and research. Contemporary Educational Psychology, 25, 92-104.

Pintrich, P.R. (2003). A motivational science perspective on the role of student motivation in learning and teaching contexts. Journal of Educational Psychology, 95, 667-686.

Pintrich, P.R., Marx, R.W., \& Boyle, R.A. (1993). Beyond cold conceptual change: The role of motivational beliefs and classroom contextual factors in the process of conceptual change. Review of Educational Research, 63, 167-199.

Pintrich, P.R., \& Schunk, D.H. (2001). Motivation in education: Theory, research, and applications $\left(2^{\text {nd }}\right.$ ed.). Englewood Cliffs, NJ: Prentice Hall.

Patricia A. Alexander es profesora y Distinguished Scholar-Teacher en el Departamento de Desarrollo Humano de la Universidad de Maryland (USA). Sus áreas de investigación incluyen las áreas siguientes: desarrollo de la pericia en dominios de conocimiento (expertise), aprendizaje basado en textos, creencias epistemológicas, persuasión y cambio conceptual.

Ha sido presidente de la División 15 (Psicología Educativa) de la Asociación Americana de Psicología (APA) y Vicepresidente de la División C (Aprendizaje e Instrucción) de la Asociación Americana de Investigación Educativa (AERA). La profesora Alexander es Fellow de la Asociación Americana de Psicología (APA) y fue Spencer Fellow de la Academia Nacional de Educación. Recientemente, un estudio publicado en 2003 por Contemporary Educational Psychology la incluía en la lista de los diez psicológos más productivos en Psicología Educativa en los últimos diez años. En 2001 recibió el premio Oscar S. Causey Award de la National Reading Conference por sus sobresalientes contribuciones a la investigación sobre la lectura y la escritura. Además ha recibido varios premios más (nacionales y universitarios) por su enseñanza. 
Hasta la fecha de su fallecimiento, co-editaba con Paul el Handbook of Educational Psychology que será publicado por Lawrence Erlaum Associates.

- Dirección postal:

Patricia A. Alexander. Department of Human Development. College of Education, University of Maryland. College Park, Maryland 20742-1131 (USA) 
Patricia Alexander

[Página dejada en blanco intencionadamente] 


\title{
La dimensión internacional de Paul R. Pintrich
}

\author{
Erik De Corte \\ Center for Instructional Psychology \& Technology, University of Leuven
}

\section{Bélgica}

erik.decorte@ped.kuleuven.ac.be

\section{Resumen}

El profesor De Corte subraya la orientación internacional de Paul Pintrich como académico. También comenta su participación en la Asociación Europea para Investigación en Aprendizaje e Instrucción (EARLI) y en la Asociación Internacional de Psicología Aplicada (IAAP) y cómo Paul trataba siempre de desarrollar lazos y vínculos entre la comunidad internacional para mejorar la investigación y diseminar el conocimiento sobre Psicología y Educación.

Palabras clave: Aprendizaje/ Psicología Educativa/ Educación 
Como muestran las contribuciones precedentes en esta parte de este monográfico, las aportaciones de Paul Pintrich al desarrollo y progreso del campo de la Psicología Educativa teórica, metodológica y empíricamente- han sido amplias desde un punto de vista cuantitativo, pero también muy sustanciales cualitativamente.

Sin embargo, además de ser un excelente académico, Paul era también una persona maravillosa con amplios intereses, una mirada crítica sobre los desarrollos sociales y un magnífico sentido del humor. Encontrarme y charlar con él fue siempre un placer para mí, y al mismo tiempo, una experiencia siempre enriquecedora.

Lo que me gustaría subrayar especialmente aquí es la orientación internacional de Paul como académico, una característica que todavía hoy no es tan obvia entre los investigadores educativos americanos. Si bien es innegable que la globalización afecta también a la comunidad académica, sin embargo, la investigación educativa americana es todavía ampliamente "provinciana". Paul, como su mentor, Bill McKeachie y otro colega y amigo fallecido, Dick Snow, era una de las notables y escasas excepciones. Es ilustrativo, en este sentido, que hasta la fecha de su muerte, era el Presidente de la División 5 (Psicología Educativa, Instruccional y escola) de la Asociación Internacional de Psicología Aplicada (IAAP). Pero la mayor evidencia de la orientación internacional de Paul fue su interés e implicación en la Asociación Europea para la Investigación en Aprendizaje e Instrucción (EARLI). Recuerdo su gran entusiasmo cuando participó por primera vez en una actividad relacionada con EARLI, el Simposium Internacional en Cambio Conceptual que tuvo lugar en Jena (Alemania) del 1 al 3 de septiembre de 1994 en la Friedrich-Schiller-University of Jena. Desde entonces, Paul se convirtió progresivamente en un participante cada vez más activo en las conferencias bianuales de EARLI y en un verdadero embajador de EARLI en los Estados Unidos. Debido a esta afinidad, se sentía muy halagado de estar invitado a dar una de las conferencias invitadas centrales en la décima conferencia de EARLI celebrada el pasado Agosto en Padua (Italia), donde debería haber hablado sobre Multiple goals and multiple pathways in the development of motivation and self-regulated learning [Múltiples metas y caminos para el desarrollo de la motivación y el aprendizaje autorregulado].

Un último ejemplo de su gran motivación hacia la internacionalización de la investigación en aprendizaje e instrucción fue su apoyo (como presidente de la División 5 de la IAAP 
y junto con el Comité Ejecutivo de EARLI) e implicación en el Seminario Internacional on “Current Issues in learning and instruction” [Temas actuales en aprendizaje e instrucción] organizado por las Universidades Cardenal Raúl Silva Henríquez y Santo Tomás, de Santiago de Chile (Chile) y celebrado en dicha ciudad entre el 2 y el 4 de Junio de 2003. Fue allí donde me encontré con Paul por última vez, completamente sano, dinámico como siempre y lleno de planes para el futuro. Por tanto, la noticia de su muerte, un mes más tarde fue al principio, increíble. Todos nosotros lo echaremos de menos como líder, pero también como un buen amigo. Nuestro homenaje a él debería ser continuar con nuestros esfuerzos para hacer investigación en aprendizaje e instrucción que persiga el alto nivel de calidad que él estableció, y que tenga como meta la mejora de la educación para todos los niños del mundo.

Erik De Corte es Profesor de Psicología Educativa de la Universidad de Lovaina (Bélgica). Su principal interés de investigación es contribuir a desarrollar teorias del aprendizaje a partir de la instrucción, y el diseño de entornos de aprendizaje poderosos, centrados en el aprendizaje, la enseñanza, la evaluación del pensamiento y la solución de problemas, especialmente en Matemáticas. Fue uno de los fundadores y primer presidente (1985-1989) de EARLI.

Fue también el editor fundador de la revista de EARLI, Learning and Instruction (19901993) y desde 1987 a 2002, editor asociado del International Journal of Educational Research. Ha co-editado (con F.E. Weinert) la International encyclopedia of developmental and instructional psychology (1996). En 1997 recibió el premio "Oeuvre for Outstanding Contributions to the Science of Learning and Instruction" de EARLI y en 2002, el premio "Award for Outstanding Contribution to Educational Psychology" de la División 5 (Educational, Instructional, and School Psychology) de la Asociación Internacional de Psicología Aplicada (IAAP). Es Fellow de la Royal Norwegian Society of Sciences and Letters, Class of Humanities, y de la Academia Europaea; también es miembro extranjero asociado de la Academia Nacional de Educación de los Estados Unidos. Actualmente es el Presidente de la Academia Internacional de Educación (1998-2006). En 2000 recibió un doctorado Honoris Causa por la Universidad Rand Afrikaans de Johannesburgo, Sudáfrica, y en 2003 por la Universidad of the Free State, Bloemfontein, Sudáfrica.. 
Erik de Corte

\section{- Dirección postal:}

Center for Instructional Psychology \& Technology (CIP\&T). Department of Educational Sciences. University of Leuven. Vesaliusstraat 2. B-3000 Leuven (Belgium).

URL: http://perswww.kuleuven.ac.be/ u0004455 


\title{
Homenaje a Paul R. Pintrich
}

\author{
Richard E. Mayer \\ Department of Psychology, University of California, Santa Barbara
}

USA

mayer@psych.ucsb.edu

\section{Resumen}

El profesor Mayer reseña cuatro contextos en el que conoció a Paul Pintrich: como un académico e investigador que contribuyó activamente al campo de la Psicología Educativa, como líder intelectual que dio forma a la Psicología Educativa, como un abogado coherente de la colaboración internacionales y como un leal defensor de su disciplina (Psicología y Educación) y de la universidad en la que realizó su doctorado y trabajó hasta su fallecimiento, la Universidad de Michigan.

Palabras clave: Psicología Educativa/ Aprendizaje / Enseñanza 
La Psicología Educativa ha perdido un querido amigo con la trágica muerte de Paul Pintrich. Paul era un líder en nuestro campo y una fuente de inspiración para aquellos que le conocíamos. Tuve el privilegio de conocer a Paul en muchos contextos: como contribuyente activo al desarrollo de nuestra disciplina, como líder, como abogado y como apoyo fiel de la institución en la que trabajaba.

$\underline{\text { Paul Pintrich como contribuyente activo al desarrollo de la Psicología Educativa. Paul }}$ Pintrich ayudó a producir la clase de investigación basada en la teoría que es esencial para el progreso científico de la Psicología Educativa. En concreto, su productividad investigadora a lo largo de todos estos años ha ayudado a clarificar el papel de los factores motivacionales, metacognitivos y actitudinales en el aprendizaje. Cualquiera que quiera escribir una explicación completa de cómo aprenden los estudiantes no podría hacerlo sin dejarse influenciar por la investigación clara y cuidadosa de Paul sobre cómo las creencias metacognitivas y la motivación influyen en el aprendizaje. He aprendido mucho del trabajo que ha publicado, escuchando sus ponencias en congresos y discutiendo su investigación con él.

$\underline{\text { Paul Pintrich como un líder intelectual que daba forma a la Psicología Educativa. }}$ Hace algunos años tuve el placer de trabajar con Paul Pintrich y algunos otros colegas en la revisión de la taxonomía de Bloom. David Krathwohl, que había ayudado a redactar la taxonomía original de 1956, nos llamó para participar en una serie de reuniones en Syracuse. Nuestro desafío intelectual era revisar el progreso en nuestro campo desde mediados de los 50 y tratar de incorporar los nuevos avances en una taxonomía revisada de los objetivos educativos. En estas reuniones, Paul fue un líder intelectual que mostró una amplia comprensión del área. Convenció al grupo de que una importante y nueva característica del aprendizaje implicaba tener en cuenta el conocimiento metacognitivo, así que, revisamos la taxonomía consecuentemente.

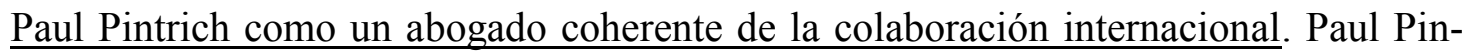
trich era un líder en la principal organización profesional de nuestro campo, la División de Psicología Educativa de la Asociación Americana de Psicología (APA), siendo su presidente y editor de su revista, Educational Psychologist. A lo largo de todo este tiempo, tuve el honor de trabajar con Paul en diversas tareas de liderazgo dentro de la División. En cada oportunidad, Paul Pintrich abogaba y apoyaba con fuerza la necesidad de colaboración internacional. Contribuyó decisivamente a que los psicólogos educativos norteamericanos se involu- 
craran más en la realización de investigación con colegas internacionales y ayudó a construir fuertes lazos de colaboración con EARLI.

Paul Pintrich como defensor de su universidad y su disciplina (Psicología y Educación). Finalmente, Paul Pintrich apoyó siempre de manera leal a su universidad, la Universidad de Michigan. Como estudiante graduado de esta misma universidad, respeto y comparto su amor por Ann Arbor. La Universidad de Michigan no podría haber pedido tener un representante y abogado mejor que Paul Pintrich.

Desde luego nuestro campo ha perdido un amigo y yo, también.

Richard E. Mayer es Profesor de Psicología en la Universidad de California, Santa Bárbara (UCSB) en la que lleva trabajando desde 1975. Obtuvo su grado de doctor por la Universidad de Michigan en 1973 y fue Visiting Assistant Professor de Psicología en la Universidad de Indiana desde 1973 a 1975. Sus intereses de investigación se centran en la psicología cognitiva y educativa. Su investigación actual tiene que ver con la intersección entre cognición, instrucción y tecnología, interesádose concretamente en el aprendizaje con multimedia y la solución de problemas.

Ha sido presidente de la División de Psicología Educativa de la Asociación Americana de Psicología (APA), ex-editor de Educational Psychologist y ex-co-editor de Instructional Science, Director del Departamento de Psicología de UCSB y ha recibido en 2000 el premio E. L. Thorndike Award por los logros alcanzados en su carrera en Psicología Educativa. Según un trabajo reciente (Contemporary Educational Psychology, vol. 28, pp. 422-430) ha sido el psicólogo educativo más productivo del período (1991-2001).

Es miembro del comité editorial de 11 de las principales revistas de Psicología Educativa. Es autor de 18 libros y más de 250 artículos y capítulos de libro, entre los que cabe destacar The Promise of Educational Psychology (1999) y Multimedia Learning (2001).

\section{- Dirección postal}

Richard E.Mayer. Department of Psychology. University of California. Santa Barbara, CA, 93106-9660 (USA) 
Richard Mayer

[Página dejada en blanco intencionadamente] 